\begin{tabular}{|c|c|}
\hline Citation & $\begin{array}{l}\text { Tuba Ayhan, Wim Dehaene and Marian Verhelst (2013) } \\
\text { A method for using sub-Nyquist sampling for ultra low-power } \\
\text { positioning systems } \\
\text { In Signal Processing Systems (SiPS), } 2013 \text { IEEE Workshop on, pp. 101-105.. }\end{array}$ \\
\hline Archived version & $\begin{array}{l}\text { Author manuscript: the content is identical to the content of the published } \\
\text { paper, but without the final typesetting by the publisher }\end{array}$ \\
\hline Published version & http://dx.doi.org/ 10.1109/SiPS.2013.6674488 \\
\hline Journal homepage & http://ieeexplore.ieee.org/Xplore/home.jsp \\
\hline Author contact & $\begin{array}{l}\text { tuba.ayhan@esat.kuleuven.be } \\
+32(0) 16321097\end{array}$ \\
\hline
\end{tabular}

(article begins on next page) 


\title{
A METHOD FOR USING SUB-NYQUIST SAMPLING FOR ULTRA LOW-POWER POSITIONING SYSTEMS
}

\author{
Tuba Ayhan ${ }^{1}$, Wim Dehaene ${ }^{1,2}$, Marian Verhelst ${ }^{1}$ \\ ${ }^{1}$ ESAT-MICAS, KU Leuven, Kasteelpark Arenberg 10, B-3001 Leuven, Belgium \\ ${ }^{2}$ IMEC, Kapeldreef 75, B-3001 Leuven, Belgium \\ \{tuba.ayhan, wim.dehaene, marian.verhelst\}@esat.kuleuven.be
}

\begin{abstract}
This paper proposes an improved Time-of-Flight (ToF) estimation algorithm that allows sub-mm position estimation and reduces the power consumption of the receiver. The former is ensured by employing a multicarrier ultra-wide band (UWB) signal. The latter is achieved through sub-Nyquist sampling allowing to decrease the rate of the analog to digital converter (A/D) and digital computational cost. This paper derives the necessary conditions to use sub-Nyquist sampling while keeping the high precision obtained from an UWB signal. These conditions involve ranging signal construction and using a phase-based ranging algorithm adapted to subNyquist sampling. The resulting algorithm achieves sub-mm ranging at $0 \mathrm{~dB}$ signal to noise ratio (SNR) by sampling a $8.76 \mu$ s long signal burst of $6 \mathrm{GHz}$ bandwidth with $343 \mathrm{MHz}$ $\mathrm{A} / \mathrm{D}$ rate. Moreover, an energy scalable receiver architecture based on this algorithm is proposed. The scalability allows dynamicaly adapting the A/D rate and power consumption to the received signal strength meeting a required estimation precision.
\end{abstract}

Index Terms - Sub-Nyquist sampling, Ultra-low power, Positioning, Time of Flight, Extremely high frequency

\section{INTRODUCTION}

Mathematically proven to be very efficient for high-precision ranging $[1,2]$, ultra-wide-band (UWB) technology has recently gained importance in accurate indoor positioning for mobile applications [3]. In [4], a range estimation algorithm achieving sub-mm precision has been proposed. This is realized through using a multicarrier UWB signal of $6 \mathrm{GHz}$ bandwidth with a carrier frequency of $60 \mathrm{GHz}$. Moreover, an unlicensed bandwidth of $6 \mathrm{GHz}$ is available at $60 \mathrm{GHz}$ band in international frequency allocation tables [5], allowing the designers freely use the band. However, processing the high bandwidth signal without information loss is also extremely challenging from an implementation point of view,

This project is funded by Flemish Agency for Innovation by Science and Technology (IWT) as SBO project OmniTrack.

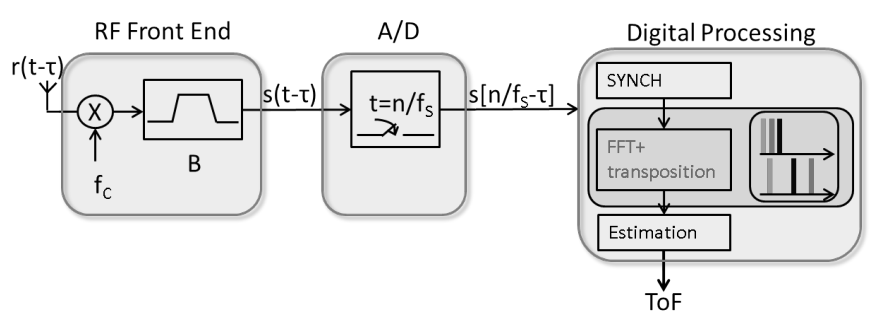

Fig. 1. A receiver for sub-Nyquist positioning systems

because Nyquist sampling rate for such systems goes up to a few Giga Hertz causing an unaffordable hardware complexity. Although A/Ds working in Giga Hertz range sampling rates are available [6], they have tight jitter and frequency offset specifications. Moreover, these fast and expensive A/Ds bring a need for processing high data rates and suffer from high power consumption. As a result, they are not suitable for mobile applications.

A low-power architecture with low complexity is needed for a sub-mm precise positioning application. To keep the circuit complexity low, a receiver which does not require a special architecture is targeted as given in Figure 1. The goal of this work is to achieve sub-mm precision with a low complexity ranging receiver and drastically reduced power consumption. In order to reduce the power consumption of the receiver, a single A/D with a rate far below the Nyquist sampling rate is used. Sub-mm precision is reached through engineering an UWB ranging signal for sub-Nyquist sampling: the $\mathrm{A} / \mathrm{D}$ rate and signal parameters are choosen in a way that, the positioning information spread over the wide bandwidth falls into a narrower band, without destructive overlap. Tuning the $\mathrm{A} / \mathrm{D}$ rate based on the targeted estimation precision and environmental conditions further reduces the power consumption at the run time. Some attempts for sub-Nyquist rate UWB positioning are discussed in the next section, however they do not fulfill the needs described above.

\subsection{Related work}

In order to cope with these complexity related problems both for the A/D side and for the process algorithm side, compressed sensing based architectures are proposed in [7] and 
the references therein. These improved architectures combine the sampling and digital processing unit in one block. In compressed sensing methods sparse or compressible signals that are sampled with sub-Nyquist rate are recovered by dedicated methods such as $l_{1}$ minimization. Compressed sensing is applied to positioning systems by fully or partially recovering the compressed sparse signals $[8,9]$. Such recovery is power consuming and inefficient compared to this work, because precise positioning in compressed domain is possible under certain conditions as will be described in the paper.

Another approach to decrease A/D rates is to use multiple RF bands requiring many low frequency channels instead of one high frequency band [10]. This method, however, increases the number of analog components in the RF receiver, causing implementation complexity, power and area overhead.

Although both compressed sensing methods and multiband $\mathrm{RF}$ receivers reduce $\mathrm{A} / \mathrm{D}$ rates, they do not significantly reduce the power consumption. Some promising positioning systems using sub-Nyquist sampling techniques have been proposed to simplify the RF front-end of the system in [11] and [12]. However, precision performance of these systems are limited to $\mathrm{cm}$ level because they cannot completely collect the positioning information spread over the ranging signal band.

This work advances the state-of-the-art, reaching an estimation precision of mm level avoiding computational complexity and power hungry A/Ds. Moreover, this technique enables adaptive systems in which the power consumption of the system is dynamically adapted to the environmental conditions and targeted accuracy.

The theoretical motivation of the new operating concept and a summary of estimation algorithm are given in section 2 . Required conditions for enabling sub-Nyquist sampling are given in Section 3. The simulation setup is introduced and results are given in Section 4. The proposed algorithm is used to build an energy scalable positioning system in Section 5, and the paper is concluded in Section 6.

\section{THEORETICAL BACKGROUND}

The performance of the position estimation is qualified with the standard deviation on estimation error, which is given by the Cramer-Rao Lower Bound (CRLB). Therefore, the theoretical analysis are done based on the CRLB, which is shown to be tight on maximum likelihood estimation simulations in the region of interest [3]. CRLB derived for the standard deviation of the ToF estimation error is

$$
\sigma^{2} \geq \frac{3}{4 \pi^{2} T \operatorname{SNR}\left(\left(f_{c}+\frac{B}{2}\right)^{3}-\left(f_{c}-\frac{B}{2}\right)^{3}\right)} .
$$

As seen from inequality (1), dominating parameters on estimation performance are the signal bandwidth (B) and the

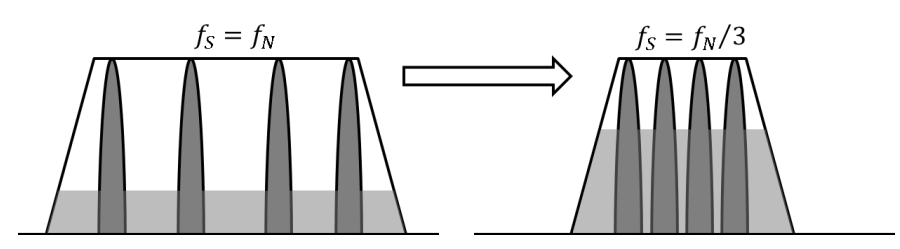

Fig. 2. Noise folding due to subsampling

carrier frequency $\left(f_{c}\right)$. The precision is quadratically proportional them, while it is linearly proportional to SNR and signal duration (T). Therefore, to improve the precision, first attempt should be increasing the bandwidth rather than signal duration or signal power. However, very high bandwidth positioning systems are not implementable due to their need of extremely high $\mathrm{A} / \mathrm{D}$ rates. In order to avoid very fast and energy hungry A/Ds, one can scale the signal bandwidth relative to the feasible A/D rates keeping the Nyquist sampling frequency $\left(f_{N}\right)$, however with a significant loss in estimation precision. As an alternative to reducing the transmitted signal bandwidth, subNyquist sampling ensuring to maintain wideband positioning information is proposed. Drawback of sub-Nyquist sampling is the SNR degradation caused by the noise folding as shown in Figure 2. With the subsampling of a multicarrier signal, the subcarriers fall into a narrower band, together with the noise in the band. For a fixed transmitted signal power, this folding decreases the SNR of the sampled signal proportional to the subsampling factor, as given in (2).

$$
\mathrm{SNR}_{\text {new }}=\mathrm{SNR}_{\mathrm{old}}-10 \times \log \left(k_{s s}\right), \text { in } \mathrm{dB}
$$

In order to argue if the SNR reduction brought by subNyquist sampling can be compensated with the high bandwidth information, two cases using Nyquist and sub-Nyquist sampling rate are compared. Figure 3 shows the theoretical ranging performances under these cases for an $A / D$ rate sweep. In case 1 , the transmitted signal bandwidth is chosen as half of the A/D rate, so that the received signal is sampled with $f_{N}$. The latter case uses the a fixed transmitted signal bandwidth of $6 \mathrm{GHz}$, but it assumes sub-sampling: SNR diminishes due to noise folding.

The difference between precision loss in case 1 and 2 increases exponentially, while the A/D rate decreases; reaching to $13 \mathrm{~dB}$ at $500 \mathrm{MHz} \mathrm{A} / \mathrm{D}$ rate. This gap between two cases means more than one order of magnitude in ranging precision: by $10 \mu$ s signal with a $0 \mathrm{~dB}$ SNR sampled at $500 \mathrm{MHz}$, proposed sub-Nyquist sampling method provides sub-mm precision, on the other hand estimation precision of only several $\mathrm{cm}$ can be achieved with the same receiver processing a narrower bandwidth signal.

This performance loss due to SNR degradation is acceptable compare to the performance loss resulting from scaling the bandwidth of the transmitted signal. Therefore, CRLB proves that sub-Nyquist sampling of a wide band signal 


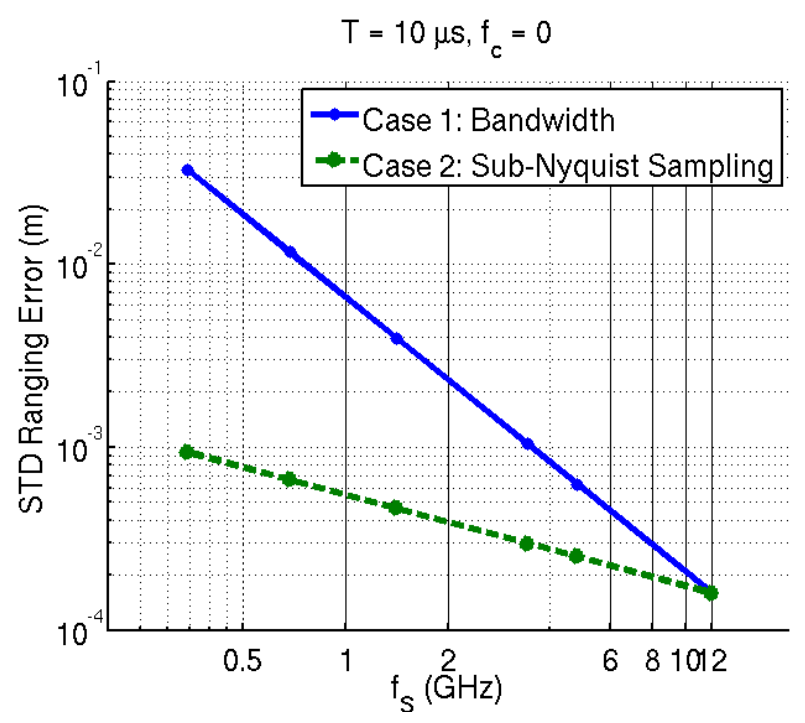

Fig. 3. Effect of low-rate A/D converters on estimation precision according to CRLB. Total signal duration is $10 \mu \mathrm{s}$, the SNR is $0 \mathrm{~dB}$ and CRLB is derived for baseband signal.

should be prefered rather than Nyquist sampling of a narrow band signal to reach very precise position estimation.

\subsection{Sub-Nyquist ToF estimation algorithm}

In order to benefit from sub-Nyquist sampling, signal parameters that are not distorted by subsampling should be used in the ToF estimation algorithm. To determine such parameters, assume a signal containing $N$ subcarriers is generated and upconverted to $\omega_{c}$ as given in (3), where $\omega_{i}$ are the subcarrier frequencies.

$$
x(t)=e^{j \omega_{c} t} \sum_{i=1}^{N} e^{j \omega_{i} t}
$$

Assuming the channel is a single path Additive White Gaussian Noise (AWGN) channel with a length of $\tau$, the received signal is

$$
r(t)=x(t-\tau)+v(t),
$$

where $\tau$ is the time of flight to be estimated and $v(t)$ is white Gaussian noise.

The received signal is downconverted, providing the input to the $\mathrm{A} / \mathrm{D}$ as

$$
s(t-\tau)=e^{-j \omega_{c} \tau} \sum_{i=1}^{N} e^{j \omega_{i}(t-\tau)}+v(t) .
$$

The signal is sampled with a frequency of $f_{s}$, so the output of the $\mathrm{A} / \mathrm{D}$ is

$$
s\left[n / f_{s}-\tau\right]=e^{-j \omega_{c} \tau} \sum_{i=1}^{N} e^{j \omega_{i}\left[n / f_{s}-\tau\right]}+v\left[n / f_{s}\right] .
$$

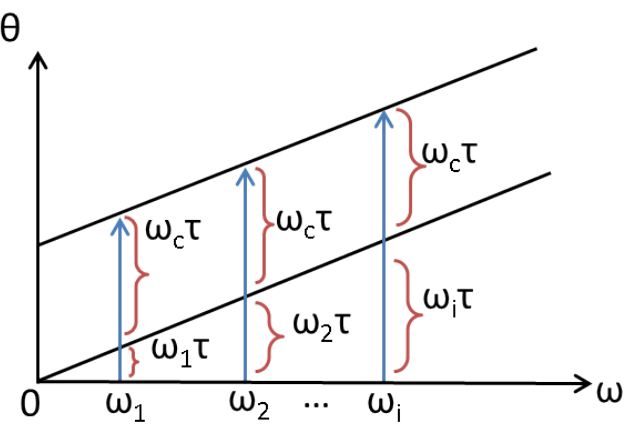

Fig. 4. A representation of frequency domain Time-of-flight estimation algorithm. The slope of the phase versus subcarrier frequency gives $\tau$.

The delay $\tau$ appears on the phase of the signal, which is not distorted by the A/D rate. Therefore, an estimation algorithm based on the phases of the carrier and the subcarriers is suitable to be used with sub-Nyquist sampling. Such an algorithm is reported in [4], the slope of the phases versus subcarrier frequencies line is estimated to calculate ToF: phase of a received subcarrier increases linearly with the ToF. After symbol synchronization, the phases of the subcarriers are used to tune ToF within one symbol period. An illustration of the estimation algorithm is given in Figure 4. For simplicity, initial phases of the subcarriers are set to 0. Following the Fourier Transform, phases of the subcarriers are sum of two terms: $\omega_{i} \tau$ and $\omega_{c} \tau$. The slope in Figure 4 is estimated using $\omega_{i}$ and estimation is further improved using $\omega_{c}$. A coarse symbol synchronization has to be performed before using phase based estimation algorithm. Since symbol synchronization is a well studied subject, it is not explained in this work. Moreover, the estimation algorithm is capable of correcting synchronization errors, if the synchronization error is less than the half of the symbol duration.

\section{ALGORITHMIC IMPLICATIONS}

According to the Nyquist theorem, for perfect data reconstruction the filtered signal should be sampled at least at Nyquist frequency: $f_{N}=2 \times B$, where $B$ is the bandwidth of the signal. On the other hand, as shown in the previous section, the phases of the subcarriers are not distorted by subNyquist sampling, if the aliasing of subcarriers is prevented.

Sub-Nyquist sampling hence does not cause positioning information loss under certain conditions. These conditions involve selection of the signal parameters and sub-Nyquist sampling rate then applying a phase-based estimation algorithm. When these conditions are fulfilled, the A/D rate can be reduced below Nyquist frequency by a subsampling factor of $k_{s s}$, where $k_{s s}=\frac{f_{N}}{f_{s}}$ and is an integer for ease of implementation. Certain signal and estimation algorithm parameters have to be chosen according to this factor $k_{s s}$. 

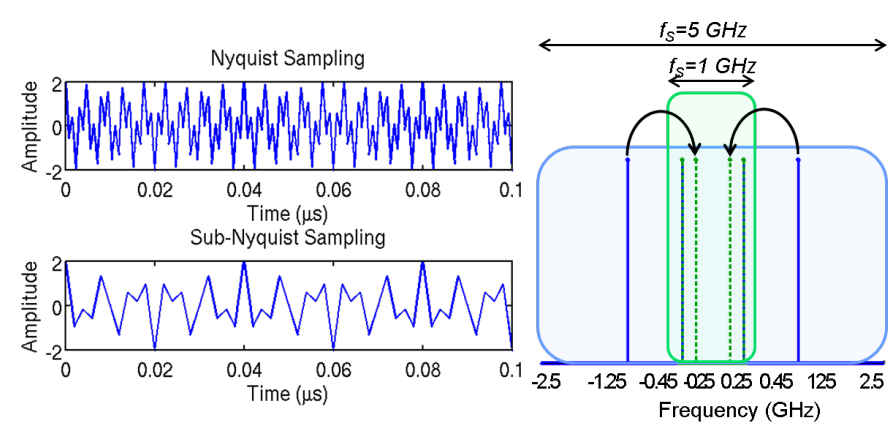

Fig. 5. Sub-Nyquist sampling of a 2 tone signal in time and frequency domains. The subsampling factor $k_{s s}$ is 5 .

\subsection{Selection of signal parameters and $k_{s s}$}

Sub-Nyquist sampling and the $k_{s s}$ factor plays a role in selecting the parameters like the subcarrier frequencies and signal duration. The subcarrier frequencies are determined such that they do not alias with each other after sampling. After subsampling, the subcarriers are aliased to a narrower band. This is visualized in Figure 5, where a signal containing two tones of $450 \mathrm{MHz}$ and $1.25 \mathrm{GHz}$ is generated with a sampling rate of $5 \mathrm{GHz}$. Then, the signal is subsampled with a $k_{s s}$ of $5\left(f_{s}=1 \mathrm{GHz}\right)$. Frequency spectra of both are shown on the right side of the figure. Sampling the $450 \mathrm{MHz}$ tone with a sampling rate of $1 \mathrm{GHz}$ does not change the position of the subcarrier in the spectrum. However, $1.25 \mathrm{GHz}$ carrier is threatened as $250 \mathrm{MHz}$ after sub-Nyquist sampling. Since these two subcarriers fall into different frequency bins, these tones are suitable to be used together for sub-Nyquist sampling with a $k_{s s}$ factor of 5 .

Given the frequencies of the tones and the A/D sampling rate, $f_{s}$, the locations of the subcarriers after sampling can be found. The tones with a frequency lower than $\frac{f_{s}}{2}$ keep their location in the spectrum of the sampled signal. For other tones, new frequency component, $f_{i}^{n}$ in the spectrum is calculated by

$$
f_{i}^{n}=\bmod \left(f_{i}^{o}, \frac{f_{s}}{2}\right)
$$

where $f_{i}^{o}$ is the original frequency of the tone $i$. Aliasing of the subcarriers to each other after sub-Nyquist sampling can be prevented using (7).

Alternatively, when the subcarriers are fixed, the $k_{s s}$ factor can be chosen, verifying the new locations of all the subcarriers. Either way, $k_{s s}$ and the subcarrier frequencies should be aligned. More than one possible $k_{s s}$ can be found for a given signal construction. This flexibility on choosing various $k_{s s}$ factors will be used to develop an energy scalable architecture in Section 5.

Another signal parameter to be settled is the signal duration. Assume, one period of the multicarrier signal, whose

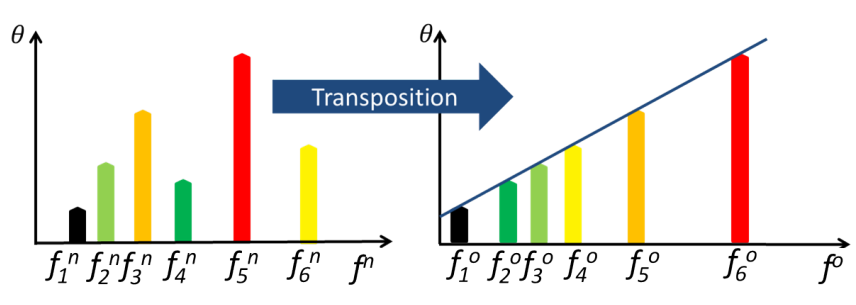

Fig. 6. Transposition of subcarriers.

subcarriers are determined by (7), is called a Short Training Symbol (STS). The received signal contains successive STSs. The number of STSs is $k_{s s}$ or multiples of $k_{s s}$ to ensure that the sufficient number of samples is used for Fourier transform.

Other signal parameters such as signal bandwidth, number of carriers and carrier frequency are not directly related to sub-Nyquist system design but affects the the precision of the estimation algorithm. Selection of them is discussed in [4].

\subsection{Frequency transpositions}

As described in the previous subsection, subcarrier locations change after sub-Nyquist sampling. On the other hand, the Fourier transform window length is maintained. The estimation algorithm is designed to process the phases of the subcarriers with the frequency values of the transmitted subcarriers. All the frequency components are recovered in a transposed order. Frequency bins transposition is found by (8), where $b_{n}$ and $b_{o}$ are the new and old bin locations respectively. Subcarrier phases calculated at new bins can be processed to estimate ToF.

$$
b_{n}=\bmod \left(b_{o} \times k_{s s}, \text { size }_{f f t}\right)
$$

Frequency transposition is visualized in Figure 6. When the FFT size is kept constant and A/D rate is reduced, frequency resolution increases. The phases of the $N$ particular tones under interest, are calculated to be used in the estimation algorithm. The calculated phases are re-transformed to the original order, so that the slope of the phases can be used to estimate $\mathrm{ToF}$ as in shown in Figure 6.

\section{SIMULATIONS}

Simulations to prove the concept of sub-Nyquist sampling in a phase-based ToF estimation algorithm for a positioning system are performed in MATLAB environment. As the transmitted signal, 16 subcarriers are generated to effectively fill the $6 \mathrm{GHz}$ bandwidth. One master subcarrier is chosen as $3 \mathrm{GHz}$ and other 15 carriers are $0.0117,0.0156,0.0234$, $0.0312,0.0469,0.0625,0.0938,0.1250,0.1875,0.25,0.375$, $0.5,0.75,1$ and $1.5 \mathrm{GHz}$. With this scheme, the length of the FFT window is 3072 samples. 


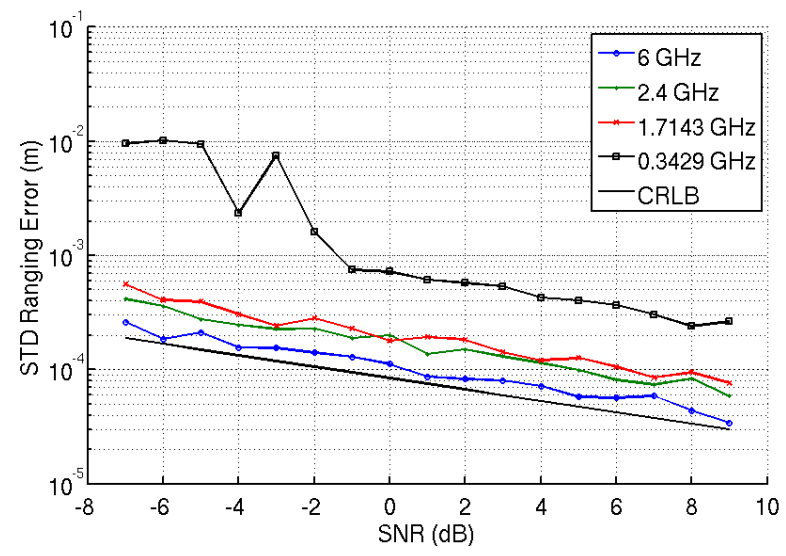

Fig. 7. Simulation results; $k_{s s}$ is $2,5,7$ and 35, resulting in 6 2.4, 1.7 and $0.3 \mathrm{GHz}$ sampling frequencies, respectively. The theoretical best accuracy for a $8.76 u$ s baseband signal is also given as CRLB.

This set of frequencies assures that the subcarriers are orthogonal and any $k_{s s}$ can be chosen except for multiples of 2 and 3. In the simulations $k_{s s}$ is chosen as an integer, so that keeping the periodicity is ensured by sending multiple STS's.

In order to maintain the generality of the proposed subNyquist sampling approach, the carrier information is not taken into account. Using a signal of $8.76 u$ s, estimation precision for $4 k_{s s}$ values (4 subsampling ratios) are simulated as given in Figure 7. For consistency, the same signal duration is used for all 4 scenarios: more STS periods are processed for lower $k_{s s}$ factors. These extra periods are used for noise averaging, by adding up the STSs. In Figure 7, estimation performance decreases with the sampling rate due to the SNR reduction.

For the 4 simulation scenarios, SNR degradation relative to the CRLB are calculated and plotted in Figure 8. For each scenario, the performance (standard deviation on ranging error) at each received SNR value is used to calculate the SNR of the sub-Nyquist sampled signal using (1). The simulation results given with the dots agree with the theoretical calculations for SNR degradation. As a result, the sampling and the processing rate can be reduced by a factor of $k_{s s}$, as far as the targeted estimation precision can be achieved in the presence of degraded SNR.

\section{ENERGY SCALABLE ARCHITECTURE}

The power consumption of the ranging system decreases with the A/D rate. Moreover the method provides advantages in the digital processing cost such as allowing a lower process rate and reduced computational cost, both reducing the power consumption of the digital processor. The latter is a result of processing less samples: the number of STSs to be used for noise averaging is decreased. Therefore, less additions are

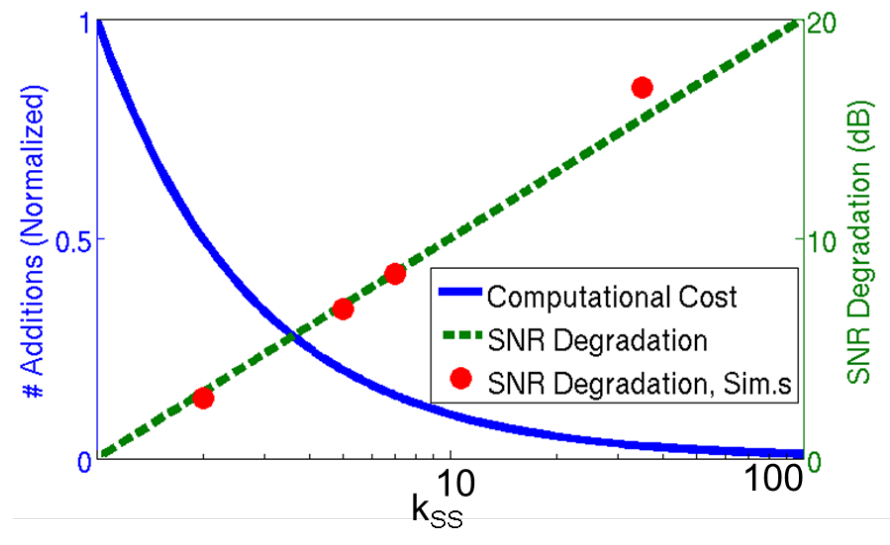

Fig. 8. Sub-Nyquist sampling reduces number of additions with a cost of SNR degradation.

performed for noise averaging. The number of additions for noise averaging is determined by number of samples to be processed;

$$
\# \text { Additions }=\frac{f_{N Y Q} \times T}{k_{s s}} .
$$

In Figure 8, number of additions used for noise averaging is given for $k_{S S}$ factors from 1 to 100 , normalized to number of additions used for Nyquist sampling case. SNR degradation due to subsampling is also shown on the same plot, where there is no SNR degradation for Nyquist sampling, $k_{S S}=1$. Sub-Nyquist sampling decreases the computational cost of the noise averaging and it does not bring additional cost to other digital processing blocks because the FFT size is maintained and the function of the original estimator algorithm is not changed. The modification to the algorithm, which is the frequency transposition is used in the algorithm by changing some constants. Therefore the overall computational cost is reduced proportional to the $k_{s s}$ which can also be scaled with the received SNR in order to meet the targeted performance criteria. Based on this result, an SNR oriented power minimization architecture for a given performance goal can be developed as a future work. In such a system, the minimum required SNR needed to achieve a certain precision can be calculated with (1), for a fixed transmitted ranging signal. Using the SNR degradation curve given in Figure 8, the subNyquist sampling factor can be adapted to the received SNR to keep the sampled signal SNR over the mentioned minimum required SNR.

A system architecture containing an SNR based $k_{s s}$ determination is given in Figure 9. In this system, the A/D rate is altered according to the received signal SNR: low, medium and high SNR regions are shown in the figure. For each region, a different $\mathrm{A} / \mathrm{D}$ rate is selected, based on the argument in Section 3.1 that more than one $k_{s s}$ factor can be found. By changing the $\mathrm{A} / \mathrm{D}$ rate at run-time, the power consumption 


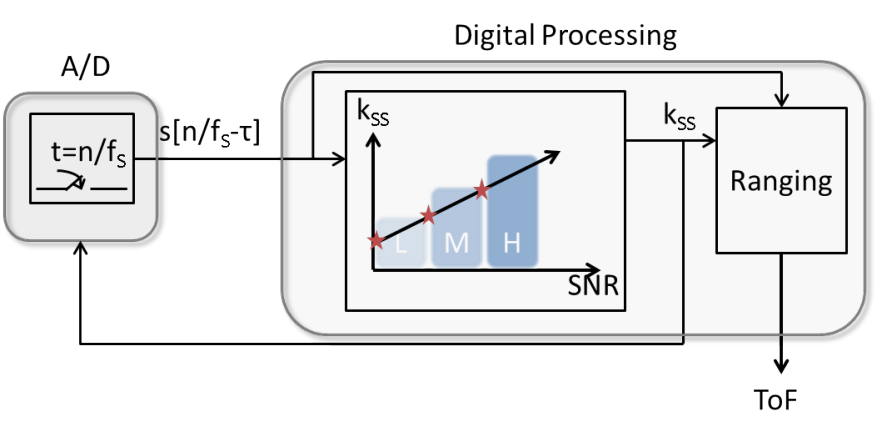

Fig. 9. A system example for selecting $k_{s s}$ according to the received SNR.

and computation load is adjusted according to the received signal quality. Therefore, an over-design caused by taking the worst case scenarios into account is prevented.

As an example, assume a precision of $0.5 \mathrm{~mm}$ is aimed with a $6 \mathrm{GHz}$ baseband ranging signal having 16 subcarriers and $8.76 \mu$ s duration as described in Section 4. To achieve this precision, the sampled signal SNR should be greater than $-12 \mathrm{~dB}$. The signal is constructed using 70 STSs which are suitable to be sub-Nyquist sampled with a factor of 2,5,7 and 35 , bringing $3,7,8.5$ and $15.4 \mathrm{~dB}$ SNR degradation, respectively. These values can be used to create 4 subgroups whose borders are determined by the received SNR values of -9 , $5,-3.5$ and $3.4 \mathrm{~dB}$. As the received SNR decreases, the A/D rate is increased to maintain the estimation performance. This way, when the subsampling factor is 35 , the number of additions for noise averaging is reduced by $97 \%$.

\section{CONCLUSION}

A mm-precise sub-Nyquist ranging algorithm is proposed and used to build an energy scalable architecture. A phase-based ToF estimation algorithm is adapted to sub-Nyquist sampling by introducing the sub-Nyquist sampling factor $k_{s s}$ and frequency transposition. The complexity of the digital processing unit and A/D rate decrease with increasing the $k_{s s}$ factor and this factor can be adjusted at run-time. By tuning this factor to trade-off between $\mathrm{A} / \mathrm{D}$ rate and estimation precision, a receiver architecture is proposed adapting the power dissipation to environment conditions and the precision goal. A periodic $8.76 \mu \mathrm{s}$ signal with a bandwidth of $6 \mathrm{GHz}$ is generated in MATLAB environment. This ranging signal contains 16 subcarriers, which are selected in accordance with various $k_{s s}$ factors. 4 of these $k_{s s}$ factors are used for simulations leading to $6 \mathrm{GHz}, 2.4 \mathrm{GHz}, 1.7 \mathrm{GHz}$ and $343 \mathrm{MHz}$ sampling rates. At $0 \mathrm{~dB}$ received SNR, a range estimation precision of $0.1 \mathrm{~mm}$ is achived with a sampling rate of $6 \mathrm{GHz}$, reducing the number of additions used for noise averaging to $50 \%$. Similarly, for the same signal strength, sampling with $343 \mathrm{MHz}$ results in $0.8 \mathrm{~mm}$ precision and uses only $97 \%$ of the additions for noise averaging. Apart from the computational cost, reduced A/D rate drastically decreases the power consumption of the ranging receiver. To the best of the author's knowledge, this is the first frequency domain range estimation utilizing sub-Nyquist sampling.

\section{REFERENCES}

[1] H. Cramér, Mathematical Methods of Statistics. Princeton, NJ: Princeton Univ. Press., 1946.

[2] C. Rao, "Information and the accuracy attainable in the estimation of statistical parameters," Bulletin of the Calcutta Mathematical Society, vol. 37, p. 8189, 1945.

[3] Z. Sahinoglu, S. Gezici, and I. Guvenc, Ultra-Wideband Positioning Systems. Cambridge Univ. Press, 2008.

[4] T. Ayhan, T. Redant, M. Verhelst, and W. Dehaene, "Towards a fast and hardware efficient sub-mm precision ranging system," in Proceedings of IEEE Workshop on Signal Processing Systems (SiPS), 2012.

[5] "FCC first report and order: In the matter of revision of part 15 of the commissions rules regarding ultra-wideband transmission systems, 2002, FCC 0215," Tech. Rep.

[6] L. Kull, T. Toifl, M. Schmatz, P. A. Francese, C. Menolfi, M. Braendli, M. Kossel, T. Morf, T. Meyer Anderson, and Y. Leblebici, "A $3.1 \mathrm{~mW} \mathrm{8b}$ 1.2GS/s Single-Channel Asynchronous SAR ADC with Alternate Comparators for Enhanced Speed in 32nm Digital SOI CMOS," in Proceedings of the 2013 International Solid-State Circuits Conference (ISSCC), San Francisco, California, 2013.

[7] M. Mishali and Y. Eldar, "Sub-nyquist sampling," Signal Processing Magazine, IEEE, vol. 28, no. 6, pp. 98124, 2011.

[8] C. Feng, S. Valaee, and Z. Tan, "Multiple target localization using compressive sensing," in Global Telecommunications Conference, 2009. GLOBECOM 2009. IEEE, 2009, pp. 1-6.

[9] S. Nikitaki and P. Tsakalides, "Localization in wireless networks based on jointly compressed sensing," in Proceedings of 19th European Signal Processing Conference (EUSIPCO 2011), 2011.

[10] R. Barrak, I. Labidi, A. Ghazel, M. Muller, and N. Samama, "RF subsampling GNSS receiver: Potential advantages and feasibility study," in Microelectronics (ICM), 2011 International Conference on, 2011.

[11] I. Maravic, M. Vetterli, and K. Ramchandran, "Channel estimation and synchronization with sub-nyquist sampling and application to ultra-wideband systems," in $I S$ CAS (5), 2004, pp. 381-384.

[12] M. Navarro Rodero, M. Najar MArton, and C. I. Casas, "Method for estimating the time of arrival in ultra wide band signals," Patent WO 2008/03 455 A, 03 27, 2008. 